\title{
A Review of Numerical Simulation and Analytical Modeling for Medical Devices Safety in MRI
}

\author{
J. Kabil', L. Belguerras', S. Trattnig' ${ }^{2}$, C. Pasquier ${ }^{1,3}$, J. Felblinger ${ }^{1,4}$, A. Missoffe $^{1}$ \\ IADI U947, INSERM, Université de Lorraine, CHRU Nancy, France \\ 2 Department of Biomedical Imaging and Image-guided Therapy, Centre of Excellence, High-Field \\ MR, Medical University of Vienna, Vienna, Austria \\ 3 Healtis, Nancy, France \\ 4 CIC-IT 1433, INSERM, CHRU Nancy, France
}

\section{Summary}

Objectives: To review past and present challenges and ongoing trends in numerical simulation for MRI (Magnetic Resonance Imaging) safety evaluation of medical devices.

Methods: A wide literature review on numerical and analytical simulation on simple or complex medical devices in MRI electromagnetic fields shows the evolutions through time and a growing concern for MRI safety over the years. Major issues and achievements are described, as well as current trends and perspectives in this research field.

Results: Numerical simulation of medical devices is constantly evolving, supported by calculation methods now well-established. Implants with simple geometry can often be simulated in a computational human model, but one issue remaining today is the experimental validation of these human models. A great concern is to assess RF heating on implants too complex to be traditionally simulated, like pacemaker leads. Thus, ongoing researches focus on alternative hybrids methods, both numerical and experimental, with for example a transfer function method. For the static field and gradient fields, analytical models can be used for dimensioning simple implants shapes, but limited for complex geometries that cannot be studied with simplifying assumptions.

Conclusions: Numerical simulation is an essential tool for MRI safety testing of medical devices. The main issues remain the accuracy of simulations compared to real life and the studies of complex devices; but as the research field is constantly evolving, some promising ideas are now under investigation to take up the challenges.

\section{Keywords}

Magnetic Resonance Imaging, safety, medical device, numerical simulation, heating, electromagnetic field

Yearb Med Inform 2016:152-8

http://dx.doi.org/10.15265/IY-2016-016

Published online November 10, 2016

\section{Introduction}

Magnetic Resonance Imaging (MRI) is an essential imaging modality, as it provides excellent resolution images without involving ionizing radiations. Three fields are used in MRI: a very high static field $B_{0}$, typically of 1.5 Tesla or 3 Tesla for clinical applications, a radiofrequency $(\mathrm{RF})$ field with an electric component of about $100 \mathrm{~V} / \mathrm{m} \mathrm{RMS}$, and gradient magnetic fields with a slope with an order of magnitude of a $\mathrm{mT} / \mathrm{m}$ and time varying with a slew rate of up to $200 \mathrm{~T} / \mathrm{m} / \mathrm{s}$.

However, with a growing number of MRI machines and MRI scans [1] as well as medical devices implantations [2], there is a need to assess the safety of MRI scans in each case. To this end, the FDA and CE provide guidelines and directives to follow according to norms as $[3,4]$. Thus, there are several different norms targeting the interactions of medical devices with each MRI magnetic field.

Medical devices with conductive parts act as antennas for radiofrequency, leading to tissue heating or dysfunction of the devices. The gradients can as well induce voltage and heating, the latter being in general less considered than RF-induced heating. When moving into the $\mathrm{B}_{0}$ field, induced currents can occur on the devices, and even when not moving the disturbance of the static field can create artifacts on the images, putting the diagnosis at risk.

One can compare simulation to experiments in order to validate a model by measuring temperature increases around a medical device. From the distribution of the electrical field and the properties of the materials, the SAR (Specific Absorption Rate) distribution in W/kg can be obtained and used as an input to a thermal simulation.

Numerical simulation is an invaluable tool for MRI safety and can help for the development of new devices. Indeed, it allows to obtain information impossible to get by experiments, like the electric field inside a human body. It is also a great way to determine the impact of different tests conditions, as this could be time-consuming and costly if done on a real MRI machine. This literature review is intended to show how numerical simulations support MRI safety testing.

\section{Numerical Methods}

Maxwell equations have to be solved to simulate the interaction of the radiofrequency electromagnetic near field with a surface or implanted medical device. For a 1.5 Tesla MRI scanner, the Larmor frequency is 64 $\mathrm{MHz}$, which corresponds to a wavelength in human tissues of about $40 \mathrm{~cm}$. Therefore, the wavelength is about the size of the studied object, and full-wave simulations need to be run meaning that no approximation on Maxwell equations can be made.

Running a simulation on a numerical human phantom is computationally expensive because it leads to a large number of discretization cells (for example up to 200 millions of cells) and a complex distribution of material of different electrical properties. The most favorable methods for this type of 
problem, in terms of memory requirements and simulation time, are the Finite Difference Time Domain [5] or Finite Integration Technique solved in the time domain. These time domain solvers require reasonable memory and simulation time compared to the frequency domain solvers (FEM: Finite Element Method and MM: Method of Moments) [6].

Moreover, these algorithms tend to be easily implemented on Graphic Processing Units (GPU) allowing a tremendous acceleration of the simulations with a hardware cost way below the cost for a cluster of CPUs. The main commercial simulation softwares available on the market providing a time domain solver are CST Microwave Studio ${ }^{\mathrm{TM}}$ (CST GmbH, Germany), Sim4Life ${ }^{\mathrm{TM}}$ (Zurich MedTech AG, Switzerland), Xfdtd ${ }^{\mathrm{TM}}$ (REMCOM Inc., USA), and FEKO ${ }^{\mathrm{TM}}$ (Altair Development SA, South Africa). ANSYS ${ }^{\mathrm{TM}}$ (ANSYS Inc., USA) and COMSOL Multiphysics $^{\mathrm{TM}}$ (COMSOL Inc., Sweden) are finite element solvers which are mainly used in the frequency domain.

One drawback of these time domain solvers is the hexahedral mesh, much less flexible than the tetrahedral mesh of a finite element solver. However, the possibility of using a conformal mesh allows to follow the strong curvature of a geometry without having to dramatically refine the mesh. This is even more important because the smaller the mesh step, the smaller the time step of the time domain solver.

After an electromagnetic simulation, the SAR distribution obtained is then scaled for each real RF pulse of the sequence and can be given as an input to the transient heat equation solver. For the magneto-static problem, for the interaction of the field with complex geometries, the most used method is the FEM. For the gradient field for which the frequency is a few $\mathrm{kHz}$, approximations can be made on the Maxwell equations and the most common method used is the FEM [7].

\section{Virtual Human Phantoms}

Human phantoms evolved tremendously since the first need for these in the 1960s [8], from an approximate geometric model to the very detailed anatomical models existing nowadays. These models are obtained from medical images from a population of diverse age, gender, or morphology, acquired from CT or MRI imaging (or even through body slices photographs, as performed in the Visible Human Project [9]). The components of the body are then segmented and a single structure in $3 \mathrm{D}$ is obtained, with all the different organs and structures in the body [10] (Figure 1) which can then be attributed corresponding dielectric properties. Many virtual human phantoms exist and are gathered in an online inventory [11].

Two main phantom types exist: voxel phantoms and surface phantoms. The first ones are voxelled, staircased models, whereas the surface models have smoother surfaces, can be meshed at one's convenience and have a high resolution that is essential when fine body structures are studied [12].

\section{Radiofrequency}

\subsection{RF Coils}

For $1.5 \mathrm{~T}$ and $3 \mathrm{~T}$ MRI scanners, the main challenge is to model the birdcage which is mainly used as the emission coil since the wide application of parallel imaging. The principle of the resonant structure is described in [13]. The issue is to tune it properly to get the wanted homogeneous mode of the resonant structure. The antenna model can be validated on phantoms by mapping of the $\mathrm{B} 1$ rotating field.

\subsection{Heating on Simple Devices}

Nowadays, many implants, active and passive, are studied for MRI safety (Figure 2). The years 2000 have been a turning point with new ASTM (American Society for

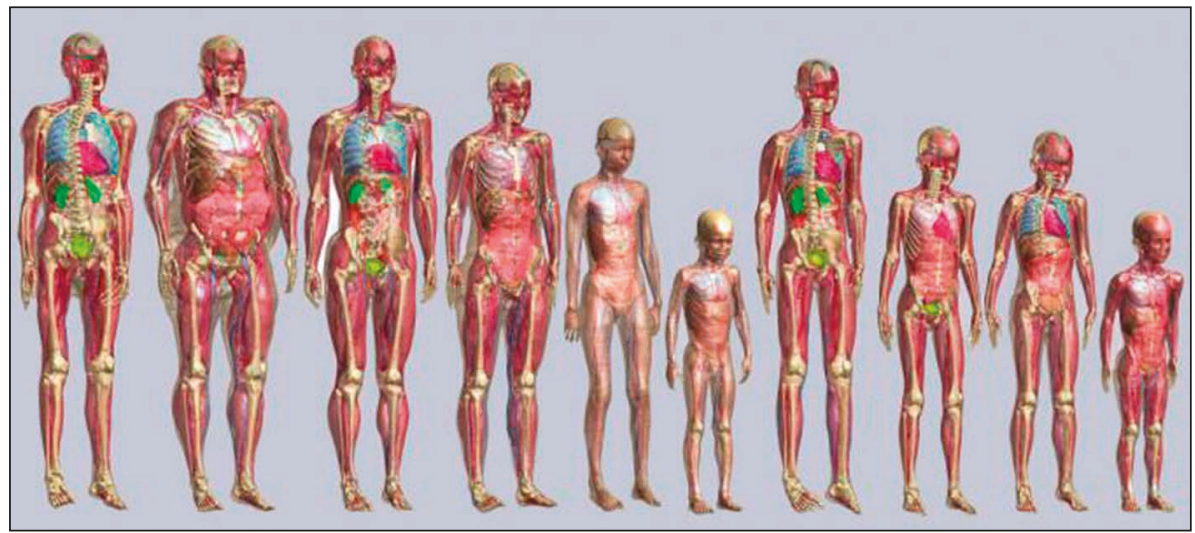

Fig. 1 Human models from the Virtual Population. @ ITIS Foundation (http://www.itis.ethz.ch/).

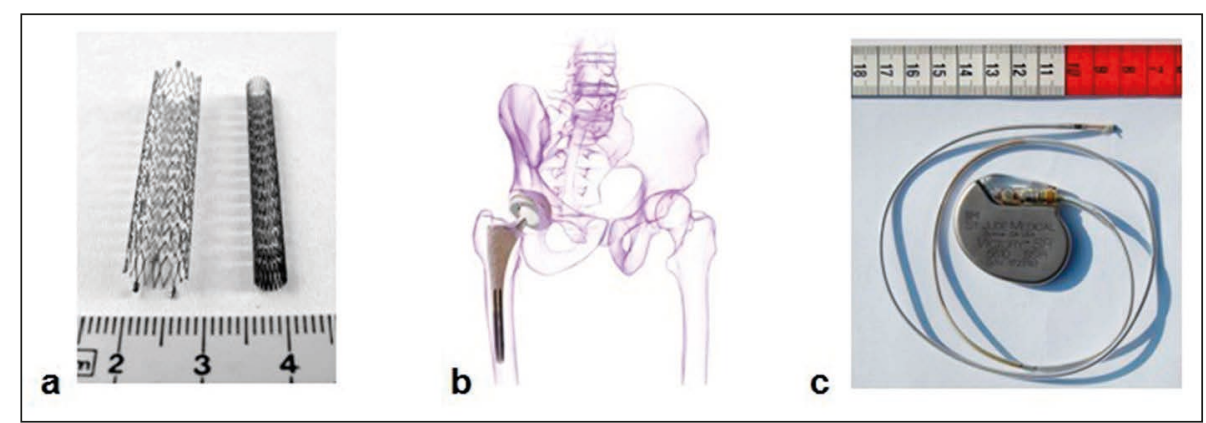

Fig. 2 a) Stents. "Stent4 fcm" by Frank C. Müller - Own work. Licensed under CC BY-SA 2.5 via Commons. b) Hip prosthesis. Adapted from "Hip Replacement" by BruceBlaus - Own work. Licensed under (C BY-SA 4.0 via Commons. c) Pacemaker. "St Jude Medical pacemaker with ruler" by Steven Fruitsmaak - self-made, removed from a deceased patient before cremation. Licensed under CC BY 3.0 via Commons. Images on Wikipedia (https://www.wikipedia.org/). 
Testing and Materials) norms published on the safety of passive device in an MRI environment [14], initiating many studies worldwide.

Several medical implants have been studied through numerical simulations, like stents [15-23]. The real CAD model can sometimes be modeled [21], showing a good correspondence between experimental and numerical results; otherwise the geometry is simplified $[15,18]$, which could lead to a different behavior regarding RF heating compared to reality. Active-MRI stents, meant to ease the follow-up of the implantation by MRI imaging, have also been simulated $[19,20]$, showing only a few Kelvin temperature increase for realistic implants. The rupture of the implant has also been discussed as it is a worst case scenario, with possible new hot spots.

Brain and head devices are also important devices regarding MRI safety, therefore aneurysm clips inside a human head model have been simulated to show SAR hotspots at 3T and 7T with different orientations of the devices [24] revealing the importance of orientation regarding RF heating. Numerous studies regarding MR safety have also been performed on orthopedic prostheses, which become very common [2] through in vitro and in vivo numerical simulations of RF-induced heating on hip prostheses [25, 26] (Figure 3 ): thus, there is a compromise to find between artifacts reduction and RF safety. Moreover, this study also revealed that the position of the implanted patient is essential, as well as the position of an implant in a phantom (as in [27-29]).

External devices have not been forgotten, with SAR studies investigating the safety of EEG metallic electrodes during MRI investigated through numerical simulation with a high resolution head model [30], concluding on dramatic averaged and peak SAR increases depending on the number of electrodes. Pin spacing and insertion depth [31] have been shown essential in external fixation devices safety assessment. A metal rod [32], cranial fixation plates [33], retinal [34] and dental [35] implants were also investigated with regard to RF heating without showing significant heating hazard in realistic conditions.

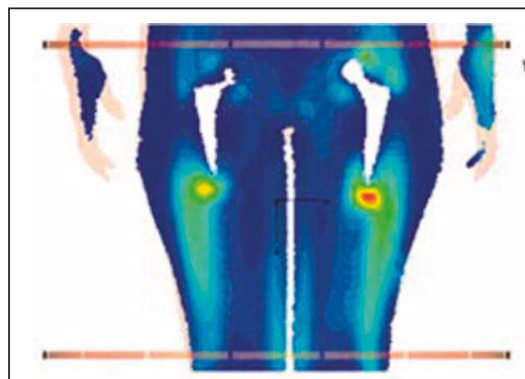

a

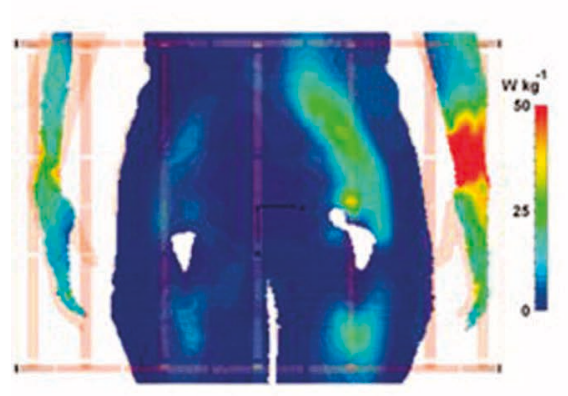

C

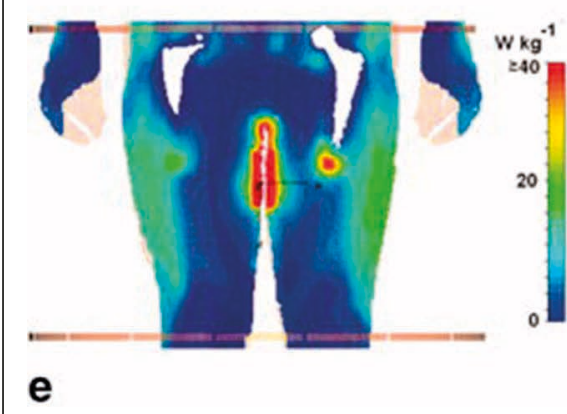

Fig. 3 SAR results in numerical simulation on a human model of hip prosthesis. J. Powell et al, Magn Reson Med 2012 Sep;68(3):960-8

Validation of RF simulations is a current topic of interest, through experimental validation in phantoms including metallic objects [36] or comparison of different solvers, BEM (Boundary Element Method), FIT (Finite Integration Technique), FDTD and FEM-BEM [37]: this last study revealed the BEM solver less accurate than the others. Interestingly, current ASTM norm on testing passive implants towards MRI RF heating [14] only recommends the use of ASTM phantoms for numerical simulations and experiments. Yet, new test methods taking additionally into account computational human models could possibly be established in the future. However, the different structures and organs of these numerical models are characterized by dielectric properties gathered in databases such as [38, 39]: as these measurements cannot always be done in vivo inside a human body, this could be a limitation and a crucial stake for the accuracy of simulations. Another issue is the validation of these human models. One way of validating the extracted geometry and different dielectric properties would be to perform experimental B1 mapping on the human body used to build the numerical model and to compare this mapping to the numerical B1 map. Temperature measurements for humans are limited to surface measurements [40], but anatomic models of a swine have been validated by comparing temperature measurements inside the brain 
to thermal simulations [41]. The importance of the details when simulating has also been recently highlighted [42] by showing that even slight changes between two versions of an anatomical model have a significant impact on numerical RF safety assessment. As there is a current problematic to quickly find the worst case amongst several device configurations, the design of experiments methods could become standards in testing devices regarding RF heating [43], and the future of simulation goes further, through the interactions of EM fields with neurons [44] and ultra high field SAR studies with or without implants [45].

\subsection{Heating on Complex Devices}

Radiofrequency of active implants is a major issue as the temperature increase can reach several tenths of degrees in a phantom for a clinical mean SAR [46]. Considering the real magnitude and phase distribution of the incident electrical field in the human body along the path of the leads of the devices considered is vitally important. The field in a phantom, even filled with gel with the mean electrical properties of the human tissues, has nothing to do with the incident field in a real human body made of considerably more heterogeneous structures with varying electrical properties. Many studies of cables [46, 47, 48] have been made with an approximately constant phase, constant amplitude incident electrical field parallel to the direction of the cable considered. This position has often been called "worst case" position but it is not considering the magnitude and phase distribution of the field. The possible phase effects of the incident field have been shown [49]. For the constant phase distribution the traditional resonance phenomena is observed; whereas for the worst phase distribution determined by simulation, the longer the cable the higher the heating (Figure 4). There is no more resonance phenomenon and a long cable that might not heat for a constant phase constant amplitude might heat for a different phase distribution.

As this worst phase distribution is not reproducible experimentally, it is necessary to determine a model to estimate this worst case heating or at least the heating for an incident field typically found in a real human body. To reach this goal, running a full-wave simulation of the device in a human body model could be a solution. But unfortunately, some implants such as pacemaker leads are made of particularly fine structure that cannot be simulated in a reasonable time.

The best approach proposed until now to estimate the heating of the lead for the incident field in the human body is the transfer function approach [50]. This transfer function allows to obtain the heating at the electrode for any incident field and consequently to get the worst case incident field phase distribution and to estimate the heating for this worst case. It is possible to measure an approximation of this transfer function by placing the lead of the device straight in gel with the mean electrical properties of human tissues $[3,14,51]$. This is only an approximation as the properties of the real tissues around the lead influence the transfer function as well as the trajectory. A calibration coefficient has to be determined to predict the actual heating. Finally, using a full-wave simulation of a human model to determine the incident field, the heating can be estimated for a lead placed in the human body. Cabot et al. [52] have studied the validity of this transfer function approach on a simple system that they could actually simulate. They compared the result from the full-wave simulation with the device in the human body and the transfer function approach. The results on the maximum 10 $\mathrm{mg}$ averaged SAR at the electrode can be more than 30\% different between the two different methods but it is still the best way up to know to estimate the in vivo heating. One possibility to improve this estimation might be to switch to a transmission line model [53] equivalent to a transfer function model with its parameters able to be adjusted considering the real tissues surrounding the lead. The same method can be applied to evaluate the RF-induced voltages on the device in vivo. [51]

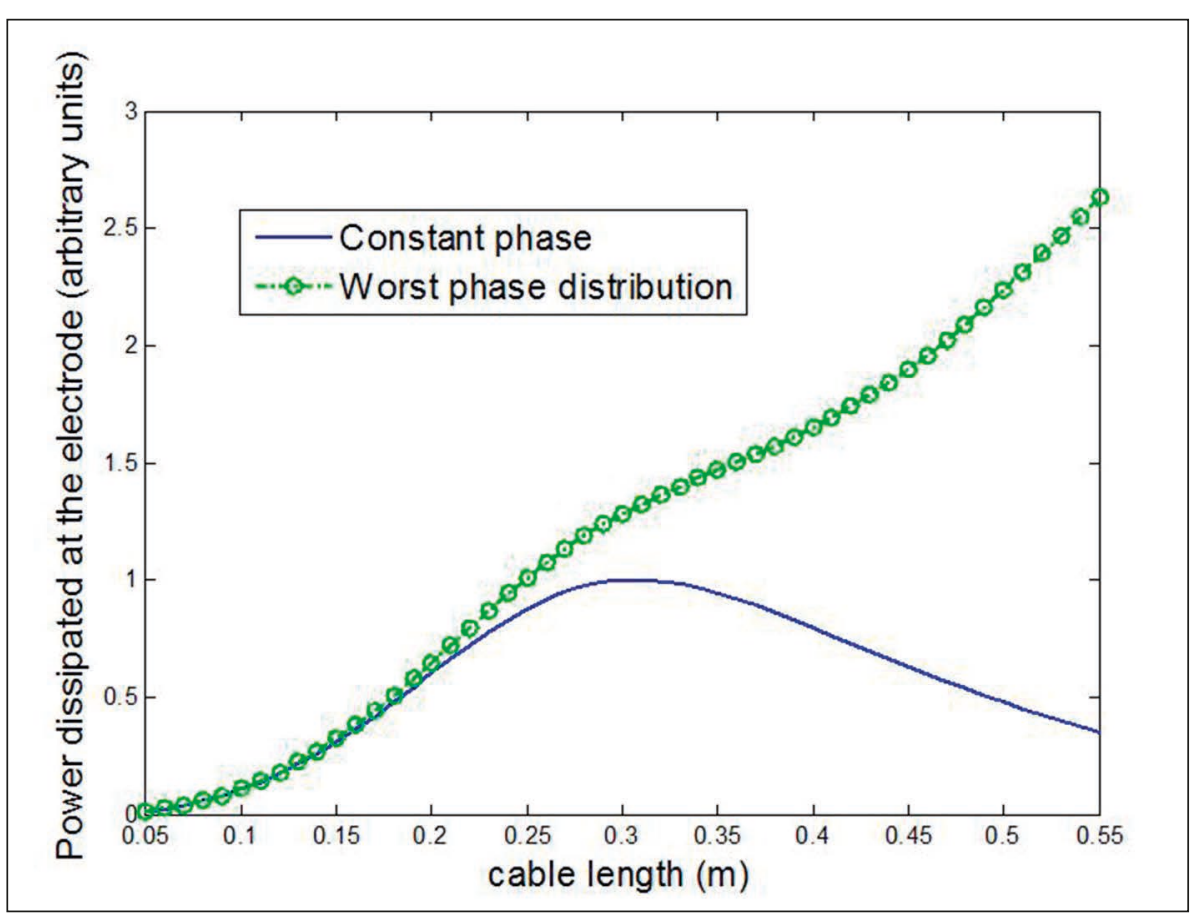

Fig. 4 Power dissipated at the electrode of a $1.5 \mathrm{~mm}$ diameter perfect electrical conducting cable insulated by $100 \mu \mathrm{m}$ of insulation of relative permittivity 4 and embedded in ASTM gel. Full blue curve corresponds to cables subjected to a constant amplitude constant phase tangential electrical field. The dashed green curve corresponds to cables subjected to constant amplitude but worst phase distribution. 


\section{Static Magnetic Field}

\subsection{Metallic Implants and $B_{0}$ Disturbance}

The presence of a metallic implant creates a disturbance of the static field, which leads to artifacts making the medical diagnosis difficult or even impossible (Figure 5).

To consider the implementation of correction techniques or magnetic susceptibility artifacts attenuation, it is essential to be able to calculate very accurately the disturbance of the magnetic field generated by the implanted metallic object placed in MRI conditions. For this purpose, analytical models can be useful tools to perform design and optimization studies on metallic objects with simple geometries $[54,55,56]$. But for more complex geometries, an accurate computation of the magnetic field is needed, using numerical methods such as FEM. These numerical methods allow to model magnetic field deformations in the diamagnetic and paramagnetic samples environment as presented in $[57,58,59]$ and they can be validated for simple geometries [57]. The numerical model was validated by comparison with the magnetic field measured by the MR gradient echo sequence in $[58,59]$.

\subsection{Motion Induced Eddy Currents for Moving Implants}

In [60, 61] Lenz effect have been studied on moving metallic implant during MRI. Indeed, a simple analytical model has been proposed to estimate the torque due to interaction between metallic implants and static magnetic field. This analytical approach is based on the Lenz' and Faraday's laws. Indeed, a rotating metal component in a magnetic field produces a current due to the change of magnetic flux with time. In addition, the changing current produces another magnetic field and this new field opposes the original field. Laplace's forces oppose the implant's movement. The developed mathematical model has been used to calculate the torques produced by the Lenz effect on the ring strengthener type of valve without considering fluid dynamics. The torque and the force calculated analytically with those obtained on a 3D-numerical model have been compared for two simple

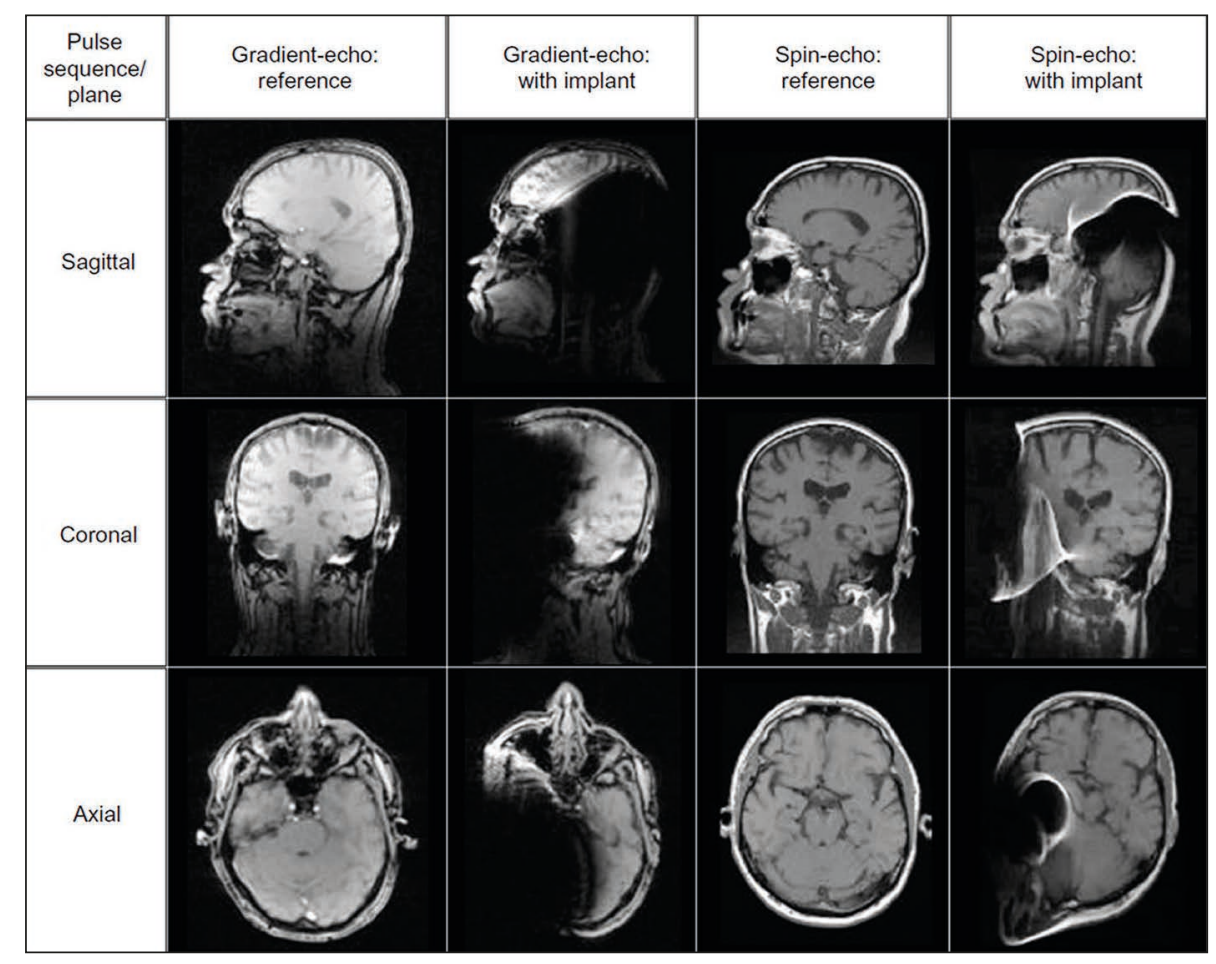

Fig. 5 MRI images in three orthogonal planes (sagittal, coronal, and axial) in a 1.5 Tesla scanner without implant (first and third column) and with implant (second and fourth column) for one spin-echo and one gradient-echo sequence, respectively. Jansson et al., Medical Devices: Evidence and Research: $8413-423,2015$.

valve shapes a disc and a ring [62], showing that the analytical approach is limited to a simple shape such as solid discs but cannot be extended to a complex shape such as a ring.

\section{Gradient Magnetic Fields}

The gradient coils of an MRI generate time varying magnetic fields with kilohertz frequency. These varying magnetic fields result in the generation of induced electric fields on metallic implantable medical devices during the MR process that results in the appearance of eddy currents which can cause heating effects. These gradient fields can also generate induced voltages on the electronics of active implanted medical devices.

\subsection{Induced Voltage}

The induced voltage generated by the time varying field can be modeled using numer- ical multi-physics software based on FEM as described in [63]. In this work, an active implant pump was studied through simulations validated by a set of experiments, and it was proved that the proposed model can be a tool for the design of critical electronic systems to ensure MRI safety.

However, these numerical methods are time-consuming. To tackle this issue, a mathematical model can allow to calculate the induced voltage for simple geometries. Simplified analytical expressions were proposed [64] to calculate the electric field induced by a linear gradient field for an active implanted medical device. In this study, it is assumed that the time-varying field is formed by infinitely long cylindrical gradient coil. The induced voltage on the implanted lead can be calculated by integrating the known electric field $\mathrm{E}$ over the length of the lead of the active implant. A comparison to experimental results showed that the induced voltage on the active implant can be determined by using these expressions with a relative error about $10 \%$. 


\subsection{Induced Heating Effect}

In [65], a hybrid model has been proposed to estimate the heating effect on patient implanted with metallic hip prostheses within an MRI-LINAC (linear particle accelerator) system. Indeed, two noncommercial codes for voxel based human models have been developed by the authors. Then, the electromagnetic field problem has been solved by using a hybrid finite element-boundary technique implemented in a GPU system. The heating effect due to the electromagnetic exposure is then estimated by using a finite element code. The simulations have been performed for a set of situations considering different possible body arrangements (Figure 6). Results showed a maximum increase of $2.5^{\circ} \mathrm{C}$.

\section{Conclusion}

MRI safety evaluations for medical devices evolved tremendously over the past decades, thanks to a global concern regarding this issue. Progresses in computer performances allowed numerical simulation to be more accessible and even to become mandatory for safety testing of devices in MRI. Nowadays, implants with a simple geometry can be simulated accurately, in a homogeneous phantom or a computational human model. Still, some questions remain about the latter: currently, the validity of human models have not been fully assessed with regard to a real human body. B1 mapping could be used to ensure the numerical and real human models are correlated enough. Moreover, human bodies being very heterogeneous and varying from one person to another, the sets of virtual models do no cover all the possibilities. Researches are relatively more recent regarding more complex implants, which are difficult or impossible to simulate because of their geometry.

Ongoing studies focus on establishing a valid mathematical model reproducing the behavior of these implants, such as pacemaker leads, towards RF induced heating. Regarding the gradients and the static field, if analytical models can be fast and accurate in most simple cases, numerical models are very helpful when simplifying assumptions cannot be used.

Safety of medical devices in MRI still involves matters of great importance for

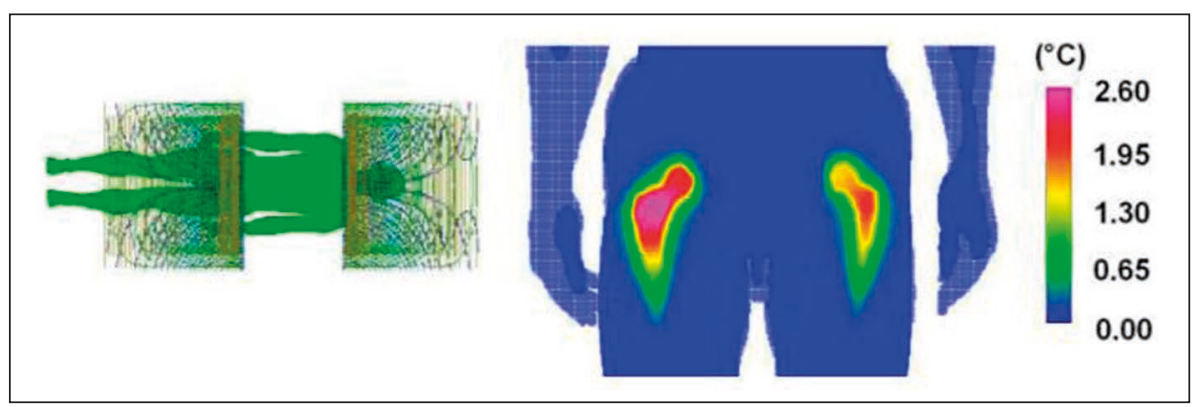

Fig. 6 Worst case when the body is in axial arrangement: body position (left) and map of temperature elevation (right). Zilberti et al., IEEE Transaction on Magnetics 2014 Nov; 50 (11).

the future, but numerical simulation has the potential to face them: it is perhaps one of the keys to safe MRI scans for everyone.

\section{Acknowledgements}

The authors thank the Région Lorraine and FEDER for funding and Pierre-André Vuissoz for technical discussion and support.

\section{References}

1. COECD/2015, OECD Data, OECD Publishing, Paris. https://data.oecd.org/

2. COECD/2015, Hip and knee replacement, OECD Publishing, Paris. DOI:10.1787/health_glance2015-36-en.

3. ISO/TS 10974:2012, Assessment of the safety of magnetic resonance imaging for patients with an active implantable medical device.

4. ASTM F2052 - 15, Standard Test Method for Measurement of Magnetically Induced Displacement Force on Medical Devices in the Magnetic Resonance Environment.

5. Yee K. Numerical solution of initial boundary value problems involving Maxwell's equations in isotropic media. IEEE Transactions on Antennas and Propagation 1996 May;14(5):302-7.

6. Ibrahim TS. Modeling the EM wave interaction with the Body and SAR. ISMRM Weekend Educational Course Syllabus 2006.

7. Büchler P, Simon A, Burger J, Ginggen A, Crivelli R, Tardy Y, et al. Safety of Active Implantable Devices During MRI Examinations: A Finite Element Analysis of an Implantable Pump. IEEE Trans Biomed Eng 2007 April;54(4).

8. Xu XG, Eckerman KF. Handbook of Anatomical Models for Radiation Dosimetry. Series in Medical Physics and Biomedical Engineering; 2009.

9. Xu XG, Chao TC, Bozkurt A. VIP-MAN: an image-based whole-body adult male model constructed from color photographs of the visible human project for multi-particle Monte Carlo calculations. Health Physics 2000 May;78(5):476-86.

10. Caon M. Voxel-based computational models of real human anatomy: a review. Radiat Environ Biophys 2004 Feb;42(4):229-35.
11. Consortium of Computational Human Phantoms (CCHP), 2011. http://www.virtualphantoms. org/phantoms.htm

12. Kim CH, Jeong JH, Yeom YS. Recent Advances in Computational Human Phantom for Monte Carlo Dose Calculation. Prog Nucl Sci Technol 2012(3):7-10.

13. Mispelter J, Lupu M, Briguet A. NMR Probeheads for Biophysical and Biomedical experiments. Imperial College Press; 2006.

14. ASTM F2182 - 11a, Standard Test Method for Measurement of Radio Frequency Induced Heating On or Near Passive Implants During Magnetic Resonance Imaging.

15. Chaudhury A, Khasnavis S, Russell M, Sarathy V. Magnetic Resonance Induced Heating in a Vascular Stent. Cornell University Library; 2007.

16. Rafiroiu D, Ciupa R, Iancu A, Lazar A, Tiseanu I. Numerical analysis of the electric field and temperature changes around carotid stents. 7th International Symposium on Advanced Topics in Electrical Engineering, 2011.

17. SPEAG Website (http://www.speag.com/), Generic stent lead simulation with Huygens Source.

18. Pawlenka M, Schaefers G. MR Safety of Implants: Numerical assessment of SAR distribution at design-simplified stents of different lengths placed inside a virtual phantom model investigated at an MR frequency of 63.9 MHz. Proc Intl Soc Mag Reson Med 2009(17).

19. Busch M, Vollmann W, Bertsch T, Wetzler R, Bornstedt A, Schnackenburg B, et al. On the heating of inductively coupled resonators (stents) during MRI examination. Magn Reson Med 2005 Oct;54(4):775-82.

20. Busch MH, Vollmann W, Schnorr J, Grönemeyer DH. Finite volume analysis of temperature effects induced by active MRI implants with cylindrical symmetry: 1 . Properly working devices. Biomed Eng Online 2005 Apr 8;4:25.

21. Camps-Raga B, Goertz W, Schaefers G, Mezape Y, Shalev A. A comparative study of numerical and experimental evaluation of RF-induced heating for an endovascular stent-graft at $1.5 \mathrm{~T}$ and $3 \mathrm{~T}$. Biomed Tech 2012; 57 (Suppl. 1).

22. Camps-Raga, B, Gortz, W, Schaefers, G, Mezape, Y, Shalev, A. Numerical and experimental evaluation of SAR hotspots for an endovascular stent graft at $1.5 \mathrm{~T}$ and 3T. International Symposium on Electro- 
magnetic Compatibility (EMC EUROPE), 2012.

23. Schaefers G, Goertz W, Noureddine Y, Koch C, Pawlenka, MJ. Magnetic Resonance (MR) safety testing of implants using numerical simulation for worst-case determination. General Assembly and Scientific Symposium, XXXth URSI, 2011.

24. Ibrahim TS, Tang L, Kangarlu A, Abraham R. Electromagnetic and Modeling Analyses of an implanted device at 3 and 7 T. J Magn Reson Imaging 2007 Nov;26(5):1362-7.

25. Hand JW, McRobbie DW. Effects of hip prostheses in situ exposed to 64 and $128 \mathrm{MHz}$ RF Field. eMagRes 2012;1(3).

26. Powell J, Papadaki A, Hand J, Hart A, McRobbie D. Numerical simulation of SAR induced around Co-Cr-Mo hip prostheses in situ exposed to RF Fields associated with 1.5 and 3T MRI body coils. Magn Reson Med 2012 Sep;68(3):960-8.

27. Liu Y, Chen J, Shellock F, Kainz W. Computational and experimental studies of orthopedic implants heating under MRI RF coils. IEEE MTT-S International Microwave Symposium Digest (MTT), 2012.

28. Liu Y. Numerical and experimental study of MRI RF signal interactions with various medical devices. PhD Dissertation, University of Houston Libraries, 2012.

29. Liu Y, Chen J, Shellock FG, Kainz W. Computational and experimental studies of an orthopedic implant: MRI-related heating at $1.5 \mathrm{~T} / 64 \mathrm{MHz}$ and $3 \mathrm{~T} / 128$ MHz. J Magn Reson Imaging 2013 Feb;37(2):491-7.

30. Angelone LM, Potthast A, Segonne F, Iwaki S, Belliveau JW, Bonmassar G. Metallic electrodes and leads in simultaneous EEG-MRI: Specific absorption rate (SAR) simulation studies. Bioelectromagnetics 2004 May;25(4):285-95.

31. Shen J, Kainz W, Qian S, Wu W, Chen J. Computational study of external fixation devices surface heating in MRI RF environment. IEEE International Symposium on Electromagnetic Compatibility (EMC), 2012

32. Nyenhuis JA, Miller CR. Calculation of Heating of Passive Implants by the RF Electromagnetic Field in MRI. XXXth URSI General Assembly and Scientific Symposium, 2011.

33. Kraff O, Wrede KH, Schoemberg T, Dammann P, Noureddine Y, Orzada S, et al. MR safety assessment of potential RF heating from cranial fixation plates at 7T. Med Phys 2013 Apr;40(4):042302.

34. Jasti S, Singh V, Lazzi G. On the modeling of the electromagnetic fields induced by MRI fields in patients with a retinal implant. IEEE Antennas and Propagation Society International Symposium, 2007.

35. Marincas C, Mada M, Rotaru H, Carpenter A, Ciupa R. The thermal effect of Radiofrequency waves near dental implants during MRI examination at 3 Tesla. Acta Electrotehnica 2013;54(1).

36. Giordano D, Zilberti L, Borsero M, Chiampi M, Bottauscio O. Experimental validation of MRI dosimetric simulations in phantoms including metallic objects. IEEE Transactions on Magnetics 2014;50(11):1-4

37. Bottauscio $\mathrm{O}$, Cassarà AM, Hand JW, Giordano D, Zilberti L, Borsero M, et al. Assessment of computational tools for MRI RF dosimetry by comparison with measurements on a laboratory phantom. Phys Med Biol 2015 Jul 21;60(14):5655-80.

38. Andreuccetti D, Fossi R, Petrucci C. An Internet resource for the calculation of the dielectric properties of body tissues in the frequency range $10 \mathrm{~Hz}-100$ GHz. Website at http://niremf.ifac.cnr.it/tissprop/. IFAC-CNR, Florence (Italy), 1997. Based on data published by C.Gabriel et al. in 1996.

39. Hasgall PA, Di Gennaro F, Baumgartner C, Neufeld E, Gosselin MC, Payne D, et al. IT'IS Database for thermal and electromagnetic parameters of biological tissues. Version 3.0, 2015 Sept, www. itis.ethz.ch/database.

40. Murbach M. EMF Risk Assessment: Exposure Assessment and Safety Considerations in MRI and other Environments. PhD Thesis, Swiss Federal Institute of Technology, Thesis No. 21514, Zurich, 2013.

41. Shrivastava D, Utecht L, Tian J, Hughes J, Vaughan JT. In Vivo Radiofrequency Heating in Swine in a 3T (123.2 MHz) Birdcage Whole Body Coil. Magn Reson Med 2014 Oct;72(4):1141-50.

42. Gosselin MC, Neufeld E, Payne D, Kuster N. Numerical simulation in virtual anatomical models: the devil is in the details. Asia-Pacific Symposium on Electromagnetic Compatibility (APEMC), 2015.

43. Abbasi M, Schaefers G, Sánchez JD, Erni D. WorstCase Analysis of RF-Induced Heating During MRI Scanning in a Generic Multi-Component Orthopedic Medical Implant Applying the Design of Experiment Method (DoE). Proc Intl Soc Mag Reson Med 2014;22.

44. Neufeld E, Oikonomidis IV, Iacono MI, Akinnagbe E, Angelone LM, Kainz W, et al. Simulation platform for coupled modeling of EM-induced neuronal dynamics and functionalized anatomical models. 7th International IEEE/EMBS Conference on Neural Engineering (NER), 2015.

45. Niendorf T, Paul K, Oezerdem C, Graessl A, Klix $\mathrm{S}$, Huelnhagen T, et al. W(h)ither human cardiac and body magnetic resonance at ultrahigh fields? Technical advances, practical considerations, applications, and clinical opportunities. NMR Biomed 2015 Feb 23.

46. Langman DA, Goldberg IB, Finn JP, Ennis DB. Pacemaker Lead Tip Heating in Abandoned and Pacemaker-Attached Leads at 1.5 Tesla MRI. J Magn Reson Imaging 2011 Feb;33(2):426-31.

47. Bottomley PA, Kumar A, Edelstein WA, Allen JM, Karmarkar PV. Designing passive MRI-safe implantable conducting leads with electrodes. Med Phys 2010 Jul;37(7):3828-43.

48. Yeung CJ, Susil RC, Atalar E. RF Safety of Wires in Interventional MRI:Using a Safety Index. Magn Reson Med 2002 Jan;47(1):187-93.

49. Yeung CJ, Susil RC, Atalar E. RF Heating Due to Conductive Wires During MRI Depends on the Phase Distribution of the Transmit Field. Magn Reson Med 2002 Dec;48(6):1096-8.

50. Park SM, Kamondetdacha R, Nyenhuis JA. Calculation of MRI-Induced Heating of an Implanted Medical Lead Wire With an Electric Field Transfer Function. J Magn Reson Imaging 2007 Nov;26(5):1278-85.

51. Feng S, Qiang R, Kainz, W, Chen J. A technique to evaluate MRI-induced Electric Fields at the ends of practical implanted lead. IEEE Transactions on microwave theory and techniques 2015 Jan; 63(1).

52. Cabot E, Zastrow E, Kuster N. Safety assessment of AIMDs under MRI Exposure: Tier3 vs. Tier4 Evaluation of Local RF-induced Heating. International Symposium on Electromagnetic Compatibility, Tokyo (EMC'14/Tokyo), 2014.
53. Missoffe A, Kabil J, Felblinger J, Pasquier C, Vuissoz PA. Determination of a transmission line model of an insulated cable for RF interaction hazard evaluation. ESMRMB Scientific Session communications, October 2015

54. Balac S, Caloz G. Induced magnetic field computations using a boundary integral formulation. Applied Numerical Mathematics 2002;41:345-67.

55. Lüdeke KM, Röschmann P, Tischler R. Susceptibility artifacts in NMR imaging. Magn Reson Imaging 1985;3(4):329-43.

56. Balac S, Benoit-Cattin H, Lamotte T, Odet C. Analytic solution to boundary integral computation of susceptibility induced magnetic field inhomogeneities. Mathematical and Computer Modelling 2004;39(4-5):437-55.

57. Olsson MB, Wirestam R, Persson BR. A computer simulation program for MR imaging: Application to RF and static magnetic field imperfections. Magn Reson Med 1995 Oct;34(4):612-7.

58. Balac S, Caloz G. Mathematical modeling and numerical simulation of magnetic susceptibility artifacts in Magnetic Resonance Imaging. Comput Methods Biomech Biomed Engin 2000;3(4):335-49.

59. Balac S. Simulation numérique des artefacts de susceptibilité magnétique en IRM. Innovation et Technologie en Biologie et Médecine (ITBM) [Numerical simulation of magnetic susceptibility artifacts in MRI. Innovation and Technology in Biology in Medicine (ITBM)] $1998 ; 19$ (5):369-79.

60. Condon B, Hadley DM. Potential MR hazard to patients with metallic heart valves: The Lenz effect. J Magn Reson Imaging 2000 Jul;12(1):171-6.

61. Robertson NM, Diaz-Gomez M, Condon B. Estimation of torque on mechanical heart valves due to magnetic resonance imaging including an estimation of the significance of the Lenz effect using a computational model. Phys Med Biol 2000 Dec;45(12):3793-807.

62. Golestanirad L, Dlala E, Wright G, Mosig JR, Graham SJ. Comprehensive analysis of Lenz effect on the artificial heart valves during magnetic resonance imaging. Progress In Electromagnetics Research 2012;128:1-17.

63. Büchler P, Simon A, Burger J, Ginggen A, Crivelli $\mathrm{R}$, Tardy Y, Luechinger R, Olsen S. Safety of Active Implantable Devices During MRI Examinations: A Finite Element Analysis of an Implantable Pump. IEEE Trans Biomed Eng 2007 April;54(4).

64. Turk EA, Kopanoglu E, Guney S, Bugdayci KE, Ider YZ, Erturk VB, et al. A Simple Analytical Expression for the Gradient Induced Potential on Active Implants During MRI. IEEE Trans Biomed Eng 2012 October;59(10).

65. Zilberti L, Bottauscio O, Chiampi M, Hand J, Lopez HS, Crozier S. Collateral Thermal Effect of MRI-LINAC Gradient Coils on Metallic Hip Prostheses. IEEE Transaction on Magnetics 2014 Nov;50(11).

\section{Correspondence to:}

Jacques Felblinger, Ph.D, Prof.

Laboratoire IADI (UL-INSERM U947)

CHRU Nancy Brabois

Rue du Morvan

54511 Vandoeuvre Cedex, France

Tel: + 33383154976

E-Mail: i.felblinger@chru-nancy.fr 